\title{
QUALIDADE DO SONO EM PACIENTES FIBROMIÁLGICOS
}

\author{
Quality of sleep in patients with fibromyalgia \\ Calidad del sueño en pacientes con fibromialgia
}

Artigo Original

\section{RESUMO}

Objetivo: Analisar a qualidade do sono em paciente fibromiálgicos, identificando seus principais hábitos noturnos e verificando os possíveis fatores que influenciam na qualidade do sono. Métodos: Pesquisa observacional, transversal, de caráter quantitativo, realizada em ambulatório de fisioterapia no período março a abril de 2012. Participaram 24 pacientes fibromiálgicos, independente do sexo e idade. Aplicou-se um questionário baseado no Pittsburgh Sleep Quality Index (PSQI) para avaliar a qualidade e hábitos noturnos. As informações foram analisadas através de estatística descritiva. Resultados: Em relação à latência do sono, apenas $1(4,2 \%)$ leva para dormir um tempo menor ou igual a 15 min e $19(79,2 \%)$ apontaram dificuldade de "não adormecer em até 30 min" por mais de 3 vezes por semana. O componente "sentir dor" e o "acordar no meio da noite" tiveram maiores influências no distúrbio do sono destes pacientes. Quanto à duração do sono, 7 (29,2\%) dormem menos que 5 horas. Sobre a eficiência do sono, 12 (50,0\%) possuíam uma eficiência do sono superior a $85 \%, 10(41,7 \%)$ possuíam eficiência do sono entre $75-84 \%$ e apenas $2(8,33 \%)$ eficiência de $65-74 \%$. Quanto ao uso de medicação para dormir, 12 (50\%) não usaram durante o mês e $12(50 \%)$ usavam entre 3 ou mais vezes durante a semana. Conclusão: Os pacientes com fibromialgia no presente estudo apresentaram um declínio na qualidade e eficiência do sono, sendo influenciados pela latência e duração do sono, presença de dor e despertar noturno.

Descritores: Sono; Fibromialgia; Fisioterapia.

\section{ABSTRACT}

Objective: To assess the quality of sleep in patients with fibromyalgia, identifying their main nocturnal habits and checking the possible factors that influence the quality of sleep. Methods: Observational cross-sectional research of quantitative approach, performed in a physical therapy outpatient center in the period from March to April 2012, with participation of 24 fibromyalgia patients, regardless of gender and age. A questionnaire based on the Pittsburgh Sleep Quality Index (PSQI) was applied to evaluate the quality and nocturnal habits. Data was analyzed through descriptive statistics. Results: As for the sleep latency, only $1(4.2 \%)$ needed an interval shorter or equal to $15 \mathrm{~min}$ to fall asleep, while $19(79.2 \%)$ pointed some difficulty in sleeping within 30 min for more than 3 times per week. The items "felling pain" and "waking up at night" had major influence on sleep disturbance in these patients. Regarding the sleep duration, 7 (29.17\%) patients sleep less than 5 hours. On the sleep efficiency, $12(50 \%)$ have values above $85 \%, 10(41.7 \%)$ have efficiency of $75-84 \%$ and only $2(8.4 \%)$ have efficiency of $65-74 \%$. Concerning the sleeping medication, $12(50 \%)$ had not used it to sleep during the month and $12(50 \%)$ used it 3 or more times during the week. Conclusion: Patients with fibromyalgia assessed in this study presented a decrease in sleep quality and efficiency, which were influenced by sleep latency and duration, occurrence of pain and nocturnal awakening.

Descriptors: Sleep; Fibromyalgia; Physical Therapy Specialty.

\author{
Florinda Freire Moro $^{(1)}$ \\ Nathália Cordeiro Leite Feitosa \\ Alexandrino $^{(1)}$ \\ Glauter José Silveira Araújo ${ }^{(1)}$ \\ José Nilson Rodrigues de \\ Menezes $^{(1)}$
}

1) Universidade de Fortaleza - UNIFOR Fortaleza (CE) - Brasil
Recebido em: 12/12/2012

Revisado em: 15/03/2013 Aceito em: 07/12/2013 


\section{RESUMEN}

Objetivo: Analizar la calidad del sueño en pacientes con fibromialgia identificando sus hábitos nocturnos principales y verificando los posibles factores que influyen en la calidad del sueño. Métodos: Investigación observacional, trasversal de carácter cuantitativo realizada en ambulatorio de fisioterapia entre marzo y abril de 2012. Participaron 24 pacientes con fibromialgia independiente del sexo y edad. Se aplicó un cuestionario basado en el Pittsburgh Sleep Quality Index (PSQI) para evaluar la calidady hábitos nocturnos. Las informaciones fueron analizadas a través de una estadistica descriptiva. Resultados: Respecto la latencia del sueño, solamente 1 (42\%) duerme menos o igual a 15 min y 19 $(79,2 \%)$ relataron dificultad de "no dormir en hasta 30 minutos" más de 3 veces a la semana. El componente "sentir dolor" y el "despertar en medio de la noche" tuvieron mayores influencias en el disturbio del sueño de estos pacientes. Respecto la duración del sueño 7 (29,2\%) duermen menos de 5 horas. Sobre la eficiencia del sueño, 12 (50,0\%) tienen la eficiencia mayor, el 85\%, 10 (41,7\%) tienen la eficiencia entre el 75-84\% y solamente 2 (8,33\%) una eficiencia del 65-74\%. Sobre el uso de medicación para dormir, 12 (50\%) no la usaron durante un mes y 12 (50\%) las usaban entre 3 o más veces a la semana. Conclusión: En el presente estudio, los pacientes con fibromialgia presentaron una disminución en la calidad y eficiencia del sueño, siendo influenciados por la latencia y duración del sueño, presencia de dolor y despertar nocturno.

Descriptores: Sueño, Fibromialgia, Fisioterapia.

\section{INTRODUÇÃO}

A fibromialgia (FM) deve ser reconhecida como um estado de saúde complexo e heterogêneo no qual há um distúrbio no processamento da dor associado a outras características secundárias. É uma das doenças reumatológicas mais frequentes, que apesar de trazer principalmente a dor musculoesquelética difusa e crônica, estes pacientes costumam queixar-se de fadiga, distúrbios do sono, rigidez matinal, parestesias de extremidade, sensação subjetiva de edema e distúrbios $\operatorname{cognitivos}^{(1)}$.

Tem sido aceito um modelo de fisiopatologia, o qual integra muitas ideias publicadas e sugere que o distúrbio primário na FM seria uma alteração em algum mecanismo central de controle da dor, que poderia resultar de uma disfunção de neurotransmissores ${ }^{(2)}$.

Com isso, o Colégio Americano de Reumatologia estabelece como critérios de diagnóstico da fibromialgia a relação do índice de dor generalizada (IDG) e a Escala de Gravidade e Sintomas (EGS), ou sintomas presentes em intensidades semelhantes por no mínimo três meses ${ }^{(3)}$.

A literatura aponta o distúrbio do sono entre uma das principais queixas em pacientes portadores de FM. Este sintoma compromete diretamente a qualidade de vida desses pacientes, podendo promover: insônia, fadiga e dor de cabeça crônica. Entre as queixas noturnas as mais comuns são: dificuldade para iniciar o sono, despertar frequente durante a noite, dificuldade para retomada do sono, sono agitado e superficial, despertar precoce; e, como consequência, o sono não reparador e cansaço podem contribuir para uma má qualidade de vida ${ }^{(4)}$.

O sono constitui-se um aspecto fundamental da vida do ser humano. Possui função restaurativa, de conservação de energia e de proteção. Sua privação pode determinar importante prejuízo em curto ou em longo prazo nas atividades diárias do paciente, causando adversidades sociais, somáticas, psicológicas ou cognitivas ${ }^{(5,6)}$.

Com isso, os distúrbios do sono no paciente com FM geram o aumento da dor e da rigidez, tornando-se importante o surgimento de novas intervenções que possam trazer a melhora na qualidade de sono, e obtendo, como consequência, a melhora na saúde e na qualidade de vida ${ }^{(4)}$.

O objetivo deste trabalho foi analisar a qualidade do sono em paciente fibromiálgicos, identificando seus principais hábitos noturnos e verificando os possíveis fatores que influenciam na qualidade do sono.

\section{MÉTODOS}

Trata-se de pesquisa observacional, transversal, de caráter quantitativo, realizada no Núcleo de Atenção Médica Integrada (NAMI) pertencente à Universidade de Fortaleza, no período de março a abril de 2012.

A amostra contemplou 24 pacientes fibromiálgicos, tendo como critério de inclusão na pesquisa os diagnosticados por reumatologista, independente de gênero e idade, e oriundos de um projeto multiprofissional de pesquisa realizado no ambulatório de fisioterapia do NAMI. Foram exclusos da pesquisa os pacientes que não foram acompanhados regularmente pelos profissionais envolvidos no projeto multiprofissional.

Por meio de uma ficha de avaliação, coletaram-se dados sociodemográficos como: idade, gênero, escolaridade e profissão.

As informações referentes à qualidade do sono e fatores que possam influenciá-lo foram baseadas no questionário validado em português, o Pittsburgh Sleep Quality Index (PSQI) $^{(7)}$.

Este questionário é composto por 19 itens, que são agrupados em 7 componentes: 1) a qualidade subjetiva do sono; 2) a latência do sono; 3) a duração do sono; 4) a eficiência habitual do sono; 5) os distúrbios do sono; 6) o uso de medicações para o sono; 7) a disfunção diurna ${ }^{(7)}$. 
A eficiência do sono é avaliada pelo tempo em que uma pessoa dorme, em relação ao tempo total que se manteve na cama durante o sono noturno e deve ser superior a $85 \%{ }^{(8)}$.

A aplicação do questionário ocorreu individualmente, sendo preenchido com a ajuda dos pesquisadores, que explicavam sobre o objetivo de cada questão, procurando facilitar seu entendimento, porém não interferindo nas respostas.

As informações foram analisadas através de estatística descritiva com porcentagens, médias e desvios padrões no programa SPSS 18.0 e expostas na forma de gráficos e tabelas.

Para a realização deste estudo, seguiram-se os princípios bioéticos previstos na Resolução 196/96 do Conselho Nacional de Saúde, que determina as normas para pesquisas em seres humanos. O projeto recebeu aprovação do Comitê de Ética da Universidade de Fortaleza (UNIFOR) - COÉTICA com o parecer n 358/2011.

\section{RESULTADOS}

Estudaram-se $23(95,8 \%)$ pacientes do gênero feminino e $1(4,2 \%)$ do masculino, com idade média de $54,5( \pm 9,3)$ anos; grau de escolaridade: 13 (54,2\%) tinham ensino médio incompleto e $11(45,8 \%)$ tinham escolaridade acima do ensino médio. Quando questionados ao tipo de ocupação desenvolvida, 7 (29,2\%) responderam ser domésticas, $4(16,7 \%)$ costureiras, $3(12,5 \%)$ aposentadas, $2(8,3 \%)$ cobradoras e $8(33,6 \%)$ realizavam outras atividades.

Em relação à qualidade do sono, $3(12 \%)$ relataram ter uma boa qualidade do sono; 7 (29\%) relataram qualidade do sono ruim e $10(42 \%)$ apresentaram distúrbios do sono.

Tabela I - Componente 2, latência do sono, do questionário Pittsburgh Sleep Quality Index (PSQI) de pacientes com fibromialgia. Fortaleza-CE, 2012.

\begin{tabular}{lcc}
\hline Componente & n & \% \\
\hline Demora a dormir & & \\
$\geq 15$ minutos & 1 & 4,2 \\
$16-30$ minutos & 11 & 45,8 \\
$31-60$ minutos & 10 & 41,7 \\
Mais de 60 minutos & 2 & 8,3
\end{tabular}

\section{Demora a dormir por mais de $30 \mathrm{~min}$ (frequência)}

Nenhuma vez

Menos de $1 \mathrm{vez} / \mathrm{semana}$

1 a 2 vezes /semana

3 vezes ou $+/$ semana

28,3

14,2

28,3

$19 \quad 79,2$

A Tabela I é referente ao componente 2, latência do sono, avaliando a quantidade de minutos que o indivíduo leva para dormir e quantas vezes por semana não consegue dormir em 30 minutos. Pode-se observar que a grande maioria, $21(87 \%)$, demorou entre 15 e 60 minutos para chegar ao sono e em relação à frequência de vezes por semana que não conseguiam dormir em 30 minutos, verificou-se que $19(79,2 \%)$ relataram uma frequência de 3 ou mais noites por semana.

O componente 3 do questionário, referente à duração do sono, indica o número de horas dormidas por noite, onde apenas $1(4,2 \%)$ dorme mais que 7 horas e $12(50 \%)$ dormem 5 horas ou menos, conforme mostra a Tabela II.

Já o componente 4, referente à eficiência do sono, composto pelo número de horas dormidas e a quantidade de horas entre deitar e levantar, identificou que $22(91,67 \%)$ possuem uma eficiência do sono acima de $74 \%$ e nenhum apresentou eficiência inferior a $65 \%$ (Tabela II).

Tabela II - Componentes 3 e 4, duração e eficiência habitual do sono, do questionário Pittsburgh Sleep Quality Index (PSQI) de pacientes com fibromialgia. Fortaleza-CE, 2012.

\begin{tabular}{lcc}
\hline Componente & n & \% \\
\hline Duração do sono & 1 & 4,1 \\
Mais que 7 horas & 11 & 45,8 \\
Entre 6-7 horas & 5 & 20,8 \\
5 horas & 7 & 29,1 \\
$\quad$ Menos que 5 horas & & \\
Eficiência habitual do sono & 12 & 50,0 \\
$\quad$ Maior que 85\% & 10 & 41,7 \\
Entre 75 - 84\% & 2 & 8,3 \\
Entre 65-74\% & 0 & 0 \\
Menor que 65\% & &
\end{tabular}

No componente 5, distúrbios do sono, encontraram-se "as dores" e "o acordar no meio da noite" como as citadas pelos pacientes.

$\mathrm{Na}$ Tabela III, observam-se os dados referentes ao componente 6 , uso de medicações para dormir, em que 12 $(50 \%)$ faziam uso entre 3 ou mais vezes durante a semana.

O componente 7 , referente à disfunção diária, que avalia a sonolência diária e o entusiasmo referentes ao ultimo mês, apresentou $14(58,3 \%)$ pacientes sem problemas em manterem-se acordados durante o dia. Porém, 5 (20,8\%) tiveram problemas em manterem-se acordados entre 3 ou mais dias por semana (Tabela III). Em relação ao entusiasmo, apenas $3(12,5 \%)$ não apresentaram problema de manter-se entusiasmados e $11(45,8 \%)$ apresentaram "grave" problema para manter o entusiasmo (Tabela III). 
Tabela III - Componentes 6 e 7, uso de medicação para dormir e disfunção durante o dia, do questionário Pittsburgh Sleep Quality Index (PSQI) de pacientes com fibromialgia. Fortaleza-CE, 2012.

\begin{tabular}{lcc}
\hline Componente & n & $\mathbf{\%}$ \\
\hline Uso de medicamento para dormir & 12 & 50,0 \\
Nenhuma vez & 0 & 0 \\
Menos de 1 vez/semana & 0 & 0 \\
1 a 2 vezes/semana & 12 & 50,0 \\
3 vezes/semana ou mais & & \\
Disfunção durante o dia & & \\
Problemas para manter-se acordado & 14 & 58,3 \\
$\quad$ Nenhuma vez & 2 & 8,3 \\
Menos de 1 vez/semana & 3 & 12,5 \\
1 a 2 vezes/semana & 5 & 20,8 \\
3 vezes/semana ou mais & & \\
Problemas para manter-se entusiasmado & 3 & 12,5 \\
$\quad$ Nenhuma & 5 & 20,8 \\
Leve & 5 & 20,8 \\
Moderado & 11 & 45,8 \\
Grave & &
\end{tabular}

\section{DISCUSSÃO}

No presente estudo procurou-se conhecer a qualidade do sono e fatores que possam influenciá-la, além dos principais hábitos noturnos de um grupo de pacientes fibromiálgicos.

Os achados sociodemográficos apresentados no atual estudo corroboram com a literatura apontando uma prevalência de fibromialgia maior em mulheres, e compreendendo uma faixa etária entre 35 e $44 \operatorname{anos}^{(2)}$.

Pesquisas demonstram que um dos principais sintomas que compõe a fibromialgia são os distúrbios no sono ${ }^{(4,9)}$. Comparando com a amostra da presente pesquisa, em que $42 \%$ dos avaliados pelo PSQI apresentaram distúrbios do sono, $29 \%$ uma qualidade do sono ruim e mesmo os $17 \%$ que relataram ter uma qualidade do sono muito boa e os $12 \%$ que relataram ter uma boa qualidade apresentaram queixas em relação ao sono, confirmam a alta prevalência desta queixa entre pacientes portadores de fibromialgia.

A insônia é o transtorno do sono mais frequente na população, representando um importante problema de saúde pública ${ }^{(10)}$. Entre os adultos, a necessidade diária de sono varia de 5 a 8 horas, em média. A maioria não se sente completamente refeito de suas necessidades de sono com menos de 7 horas por dia, embora as demandas socioculturais habitualmente os impeçam de dormir menos do que sua necessidade endógena ${ }^{(11)}$. Boa parte dos indivíduos analisados no presente estudo apresentaram duração do sono entre 6 e 7 horas e até mesmo menor que 5 horas por noite.

As principais queixas relacionadas aos distúrbios do sono são a dificuldade de iniciar o sono, múltiplos despertares noturnos com dificuldade para voltar a dormir, despertar precoce, além de sonolência/fadiga persistente durante o $\mathrm{dia}^{(5)}$. Dentre as manifestações da fibromialgia, os distúrbios do sono representam um importante aspecto a ser analisado. São frequentes, em especial, os eventos de fragmentação do sono, tais como o aumento do número de despertares breve ${ }^{(12)}$. No presente trabalho ficou evidente a frequência do acordar durante a noite, principalmente por conta das dores, ou até mesmo para ir ao banheiro e pesadelos foram citados como fatores que influenciaram na duração e eficiência do sono.

Pacientes fibromiágicos apresentaram menor tempo total de eficiência do sono; uma alta frequência de despertares a noite ocasionados pela dor; longo tempo para iniciar o sono, o que caracteriza sono não restaurador ${ }^{(11)}$. Esses resultados são similares aos que foram encontrados na presente pesquisa, no qual a dor e o acordar no meio da noite tiveram bastante influência nos distúrbios do sono nos pacientes avaliados.

A literatura aponta as alterações no padrão de sono de indivíduos com fibromialgia e diminuição da eficiência do sono evidenciados desde a década de 70 , que já vêm relatando a diminuição da eficiência do sono e do tempo total de sono nesses pacientes ${ }^{(12)}$. No presente estudo foi visto que metade (12) dos indivíduos teve uma eficiência do sono maior que $85 \%$ e a outra metade (12) abaixo de $85 \%$.

O somatório dos componentes avaliados aponta um déficit na eficiência do sono e possíveis repercussões na qualidade de vida, além de repercussões fisiológicas na saúde dos indivíduos. No caso da fibromialgia, isso gera um ciclo vicioso, onde o sono não reparador tende a aumentar a fadiga muscular e o aumento da dor, que por sua vez vai interferir novamente na qualidade do sono.

\section{CONCLUSÃO}

O presente estudo identificou, neste grupo de pacientes com fibromilagia, um declínio na qualidade e eficiência do sono, sendo influenciados pela latência e duração do sono, presença de dor e despertar noturno.

\section{REFERÊNCIAS}

1. Heymann RE, Paiva EDS, Helfenstein Junior M, Pollak DF, Martinez JE, Provenza JR, et al. Consenso brasileiro de tratamento da fibromialgia. Rev Bras Reumatol. 2010;50(1):56-66. 
2. Helfenstein Junior M, Goldenfun MA, Siena CAF. Fibromialgia: aspectos clínicos e ocupacionais. Rev Assoc Med Bras. 2012;58(3):358-65.

3. Wolf F, Clauw DJ, Fitzcharles MA. The American College of Rheumatology preliminary diagnostic criteria for fibromyalgia and measurement of symptom severity. Arthristis Care Res. 2010;62(5):600-10.

4. Gui M, Pedroni CR, Rossini S, Reimão R, Barbosa CMR. Distúrbios do sono em pacientes com fibromialgia. Neurobiologia. 2010;73(1):175-82.

5. Chokroverty S. Overview of sleep \& sleep disorders. Indian J Med Res. 2010;131:126-140.

6. Neves GSML, Giorelli AS, Florido P, Gomes M da Mota. Transtornos do sono: visão geral. Rev Bras Neurol. 2013;49(2):57-71.

7. Bertolazi AN. Tradução, adaptação cultural e validação de dois instrumentos de avaliação do sono. Escala de sonolência de Epworth e índice de qualidade de sono de pittsburgh [dissertação]. Rio Grande do Sul: Universidade Federal Rio Grande do Sul; 2008.

8. Chellappa SL, Araújo JF. O sono e os transtornos do sono na depressão. Rev Psiquiatr Clín. 2007;34(6):2859.
9. Steffens RAK, Liz CM, Viana MS, Brandt R, Oliveira LGA, Andrade A. Praticar caminhada melhora a qualidade do sono e os estados de humor em mulheres com síndrome da fibromialgia. Rev Dor. 2011;12(4):327-31.

10. Gulyani S, Salas RE, Gamaldo CE. Sleep medicine pharmacotherapeutics overview: today, tomorrow, and the future: part 1: insomnia and circadian rhythm disorders. Chest. 2012;142(6):1659-68.

11. Ferreira KASL, Mello DS, Dias AF, Teixeira MJ. Alterações na qualidade do sono prejudicam a qualidade de vida de pacientes com dor crônica. Rev Dor. 2006;7(4):892-904.

12. Campos RMS, Silva A, Queiroz SS, Mônico Neto M, Roizenblatt S, Tufik S, et al. Fibromialgia: nível de atividade física e qualidade do sono. Motriz Rev Educ Fís. 2011;17(3):468-76.

\section{Endereço para correspondência:}

Jose Nilson Rodrigues de Menezes

Universidade de Fortaleza

Curso de Fisioterapia

Av. Washington Soares, 1321

Bairro: Edson Queiroz

CEP: 60.811-905 - Fortaleza - CE - Brasil

E-mail: nilsonmenezes@unifor.br 